\title{
An SSM-Based Approach to Implement a Dynamic Performance Management System
}

\author{
Roberto da Piedade Francisco and Américo Azevedo \\ INESC Porto \& Faculdade de Engenharia da Universidade do Porto, \\ Rua Doutor Roberto Frias S/N \\ 4200-465 Porto, Portugal \\ \{roberto.piedade, ala\} @fe.up.pt
}

\begin{abstract}
This paper underlines how the use of Soft Systems Methodology (SSM) for an efficient planning, implementation and monitoring of a dynamic performance management system supported by a conceptual scheme that enables a conscious and prepared implementation, can provide instances of performance of a collaborative network, and also promote alignment among the partners. A systematic way to implement it and a review on two practical applications in Brazilian collaborative networks of SMEs are also presented.
\end{abstract}

Keywords: Collaborative Networks, Soft Systems Methodology, Performance Management.

\section{Introduction}

The establishment of Collaborative Networks $(\mathrm{CN})$ aims at reducing uncertainty and increasing competitiveness. This subject is increasingly acknowledged by academics and practitioners as a new paradigm that involves interoperation of distinct organisational systems that search for effectiveness, aggregating skills and resources of network's participants. Monitoring the CN's performance is advisable in order to understand how it is possible to manage this new environment and thus assist decisionmakers in achieving the goals that motivated the creation of the $\mathrm{CN}$.

The line of thought in this ongoing research implies that in dynamic environments, an organisation must obtain performance measurement outcomes and use them to obtain the results of operations, and to create actions that solve problems through available systematic practices in order to find an appropriate management tools.

This paper intends to highlight some issues regarding performance management in collaborative networks and encourage the use of the Soft Systems Methodology (SSM) in order to apply an action research to build a Performance Management System (PMS). After the introduction of relevant aspects, this document is organized as follows: the second section presents some definitions and explanations on performance management. In the third section an overview about SSM is provided. Then, the fourth section addresses the conceptual approach to support the creation of a dynamic Collaborative Network Performance Management System (CNPMS), and finally some conclusions are presented in fifth section. 


\section{Performance Management}

Performance management systems are yet an important management decision support tool that represents a prerequisite to assure effective network operations towards operational excellence. Nevertheless, defining an appropriate set of categories of performance measures that meet the needs of a particular network represents a critical step towards the establishing of an effective performance management system. In order to contribute to support the $\mathrm{CN}$ decision-makers it is necessary to know how to define, configure, and implement an effective CNPMS.

\subsection{Measurement and Management of Performance}

Busi \& Bititci [1] argue that the measure of performance is just the practical and technical instrument of performance management, which is something broader. Measuring performance helps monitor performance and identify processes and/or areas that need attention. According to Amaratunga \& Baldry [1], performance management is defined as "the use of performance measurement information to effect positive change in organisational culture, systems and processes by helping to set agreed-upon performance goals, allocating and prioritising resources, informing managers to either confirm or change current policy or programme directions to meet those goals". The authors explore these baselines in order to facilitate the transition from performance measurement to performance management.

Lardenoij, Raaij \& Weele [2] define performance measurement (referring Neely) as the "process of quantifying the efficiency and effectiveness of actions in order to compare results against expectations with the intent to motivate, guide and improve decision making", and define performance measurement system as "the set of metrics used to quantify the efficiency and effectiveness of actions, and the corresponding guidelines for linking these metrics to strategy and improvement". For an organization to make effective use of the performance measurement outcomes, it should be able to make the transition from measurement to management.

There are some specific performance management systems used in CNs that are found, applied by practitioners and adapted to each specific application, such as SCOR model [3], GPM-SME [4], PMS-EVE [5], and others specifically about virtual organizations, such as VOPM [6]. At the same time, there are some conceptual models such as a SCOR based model [7] and CNPMS [8]. Each of the models consists of gathering some concepts in order to build a framework that can support decisionmakers so that they can achieve the specifics purposes or strategies and identify new challenges.

According to Supply-Chain Council [3], the Supply Chain Operations Referencemodel (SCOR model) it is "a unique framework that links business process, metrics, best practices and technology features into a unified structure to support communication among supply chain partners and to improve the effectiveness of supply chain management and related supply chain improvement activities". This model tries to understand the enterprises' processes and the necessary reconfiguration, evaluating the performance of the internal processes and redefining the targets. At the same time, it proposes best practices to improve performance and imposes an alignment standard to features and functionality. 


\section{Soft Systems Methodology (SSM): An Overview}

The SSM is a systematic way of using a structured approach that basically intends to understand a problem, build a conceptual model, find feasible and desirable changes and implement them.

\subsection{SSM as a Learning Process}

Proposed by Checkland [9], the SSM was developed in order to handle organizational problems. It is used in order to respond to symptoms caused by underlying problems, or when that problem is not yet understood [10].

According to Graelm, Graelm \& Erdmann [11], a methodology is often confused with a method or technique when there is a situation that should be described or solved. Methods describe what to do and their results are previously defined expectations. Furthermore, methodologies are considerations that require structured approaches and analyses. It helps decision-makers understand the problems of the real world by comparing the perception of people, through the construction of theoretical models in order to facilitate the learning process.

Ferrari et al. [12] explain that SSM it is "a methodology that tries to analyze, under a systematic focus, a real organizational problem, extracting from this analysis actions for the improvement of the real world".

\subsection{SSM as a Systematic Practice Approach}

In order to better understand the complexities involving the existing organizations, there is a tendency to change the traditional systemic approach, or "hard-systems", in which the control of physical systems is based on predefined goals, for a "soft systems" that emphasizes the features found in complex systems of human relations.

Ferrari et al. [12] point out that "systemic practices are applications of systemic thought to start and to guide the actions of the real world". In this context, it is necessary find an adequate systematic practice to solve problems.

According to Checkland [9], the SSM contains a reasonable explanation for a scientific application and it is divided into seven distinct stages (see Figure 1):

1. The problem situation is structured and the key players as well the processes are defined in order to start the analysis or review;

2. The organizational structure and processes, as well the specific management and hardware technologies, are reviewed using techniques that can illustrate the problem situation and select the information to support the analysis;

3. Relevant systems are addressed using root definitions in order to express the central purpose of the chosen activity system and also using the CATWOE technique where some elements are used in order to understand the analysis of root definition sentences, and then originates transformations;

4. Conceptual models are built in order to be a model of human thought pattern that strictly conforms to the root definition using a minimum set of activities that can be drawn by applying system thinking;

5. Compares conceptual models with reality. Back to the real world, thinking on the adopted line. The conceptual models (stage 4) must be compared with real world expressions (stage 2); 
6. Implement 'feasible and desirable' developments or/and changes that must be identified and discussed so that they can be put in action in the next stage;

7. Action to improve the problem situation in order to prepare solutions and define how to implement them in step 6.

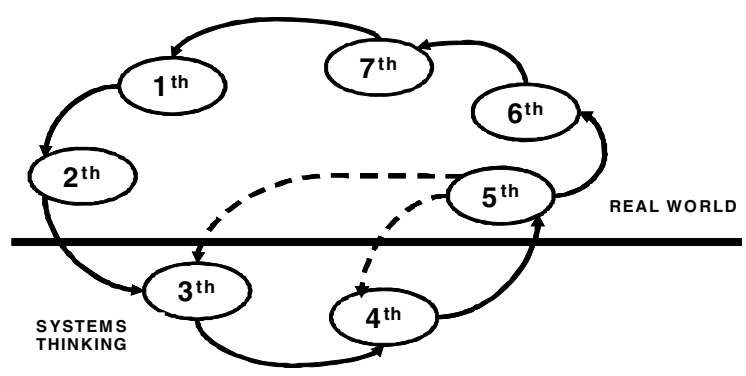

Fig. 1. The seven-stage of Soft Systems Methodology model

According to Bergvall-Kareborn et al. [13], the CATWOE technique is a combination of intuition, experience and willingness to deal with formal systems thinking. The main objective is to understand properly the meaning of CATWOE elements and their inter-relationship in order to improve the analysis. These elements are: customer, actor, transformation process, weltanschauung (world view), owner, and environmental constraints.

\section{On the Design of PMS for Collaborative Network Environment}

The PMS is still an important tool to support decision making. In fact, it is a prerequisite to ensure effective operations among partners. At the same time, it can help organizations provide instances of performance, and simultaneously lead to the alignment of CNs' participants.

Indeed, the emerging model of collaborative business requires appropriate infrastructure and technology support, as well as solutions in performance management that can ensure the alignment of strategic objectives among business partners in a collaborative networked business environment. So, it is extremely important that the $\mathrm{CN}$ is able to develop a structured way to design its PMS. This is a prudent way of finding appropriate management tools in addition to solutions for appropriate performance measurement. Thus, it seems that the use of SSM can develop an adequate methodology in order to find the necessary requirements for the definition of a performance management framework, as well as methods to implement it in practical applications.

\subsection{Using SSM Approach to Implement a PMS}

The purpose of using this methodology is to analyze how it is possible to configure and implement an effective PMS in a CN. Practical applications presented below 
showed that, when the construction of PMS framework occurs, there is a need to use scientifically consistent systematic, enabling and feasible solutions on trustworthy aspects regarding the definition of indicators, strategies and common goals, monitoring results, among others.

The implementation of a PMS in a $\mathrm{CN}$ is the problem situation that we must deal with. The use of the SSM enables a simple, adaptable and flexible methodology so that the decision-makers of the $\mathrm{CN}$ can use a sequence of steps (systematic) and thus bring together the interests and participation of each party in the design and implementation of CNPMS.

Thus, following the SSM assumptions, on the steps to create CNPMS are defined as follows:

1. Know the strategies and inter-operations of $\mathrm{CN}$, and also the skills and expertise of partners in performance measurement and management;

2. Undertake effective analysis about the benefits for each partner of the $\mathrm{CN}$, in accordance with their strategic objectives and purposes;

3. Choose a set of indicators that can translate the strategic and measurable objectives, and then consider an appropriate structure for the CNPMS;

4. Continuously check whether the alignment is maintained through performance feedback after the assessment of decision-makers;

5. Continuously monitor the performance of $\mathrm{CN}$ and check if the current results are compatible with those originally proposed;

6. In the event of poor performance or difficulty in achieving the goals and strategies, the processes of inter-operations must be reconfigured;

7. Check in time if the intentions and opportunities that promote the creation of the NC are still valid.

\subsection{PMS Framework}

According to Busi \& Bititci [1], through a compilation of Adair et. al, Amaratunga \& Baldry and Wagooner et. al works, support performance management systems should include the following "key elements":

- A structured methodology to design the PMS;

- A structured management-process for using performance measurement information to help make decisions, set performance goals, allocate resources, inform management, and report successes;

- A set of requirements specifications of the necessary electronic tools for the gathering, processing and analysis of data;

- Theoretical guidelines on how to manage through performance management systems are used to apply the information and knowledge arising from performance measurement systems; and

- A review process to ensure that measures are constantly updated to reflect changes in strategy and/or market conditions.

The framework proposed by Azevedo \& Francisco [8] is based on two main layers (see Figure 2): data and information layer, and functionality layer. 


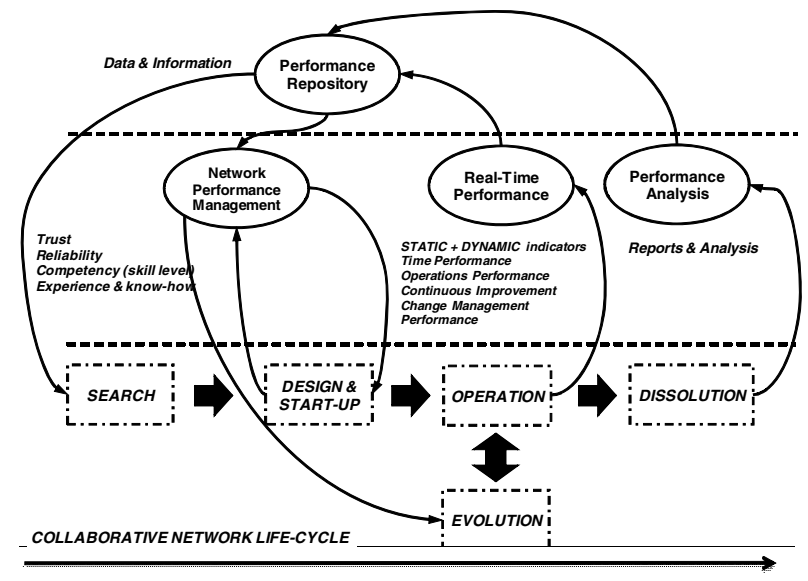

Fig. 2. Collaborative Network Performance Management System (CNPMS)

The first layer is related to data acquisition and repository management. The view of a performance repository of partners intends to provide trustworthy information about reliability, competences (skill level), experience and know-how. It is possible to achieve a time gain in the Search and in the Design phases.

The second layer comprises three main performance functionalities: network performance management to support the Design \& Start-up phase, and also the Evolution Phase; real-time performance management to measure the outputs, solve emerging problems and formulate improvements during the Operation and Evolution phases, and performance analyses in order to get to know and understand the performance and knowledge reached during the life-cycle. To appropriately manage the Operation phase, there is a set of indicators in a specific chosen PMS framework through static and dynamic indicators that enable the improvement of the time factor (agility), operation performance, and provide sustainability for a continuous improvement and change management. This matter also has an implication on the Evolution phase. The static indicators provide the results of the operations, and the dynamic indicators can be set to create actions that enable problem solving.

Then, it is realized a performance analysis in order to understand if the $\mathrm{CN}$ has reached it goals and also to obtain a memory regarding their performance and to draw knowledge from the analysis itself. This is mandatory in the Dissolution phase.

\subsection{Practical Applications}

An explanatory case study was used to validate and prove the proposed system applied in two Brazilian collaborative networks in SMEs. The results encouraged the authors to further develop the system.

Application 1. LogVale Logistics is a small company that offers logistics operations management, seeking to decrease cost solutions for their customers. In 2007, the authors conducted an experiment where they implemented the CNPMS model in order to carry out an action research. In the beginning, there was particular concern with 
issues of trust, relationships and interoperability, which were considered to be the problem situations. The base of the CN is set up by this logistics company, which supplies products for a sales company of motorcycle (Motovale), assisted by a consulting company (Profit Able) and ICT services (E-Solution) acting as a general network topology that considers the proposition of Afsarmanesh, Marik \& Camarinha-Matos [14] about a temporary partnership that is established in order to explore market opportunities on the short term.

The three initial steps (see 4.1) of the conceptual schema have been implemented in view of the need to prove (partially) the viability of the methodology. The aspects related to the strategy and skills were reviewed. The partners realized the benefits that could stem from the scope of its strategic objectives and were motivated to integrate operations. Thus, the internal processes and the inter-operations have been properly defined and integrated. After the implementation of the scheme, managers could verify the alignment among partners, improve the CNPMS and reconfigure processes and inter-operations that registered low performance. Thus, it was possible to validate the original purposes. Currently (2009), the $\mathrm{CN}$ is in its Dissolution phase. Step 6 and 7 are still being analyzed.

Application 2. In 2009, the companies Cepalgo Films (plastic films), Cepalgo Embalagens (plastic packs), and GSA (candy and snacks) intends to observe the interoperation among them working in a networked environment as a chain topology. This partnership was consolidated because the companies are controlled by the same stakeholders. Nevertheless, each one of them has their specific products and commercializes them to other clients, each one in their respective area. The Cepalgo Films produces coextrusion plastic films and supplies about $50 \%$ (internal production) to Cepalgo Embalagens. On the other hand, this company supplies about 40\% to GSA. The network strategy is to reduce that dependency more and more and look beyond the company's boundaries. Each one has autonomy (budget, infrastructure, market) despite not having a collaborative production planning and, therefore, there are no gains in synergy. The two initial steps are concluded yet.

Considering the proposition of Afsarmanesh, Marik \& Camarinha-Matos [14], the network topology is a dynamic project-based partnership without a dominant participant. The problem situation that has to be solved is: how can we improve the logistics performance, as well as the quality of products, processes and people?

\section{Conclusions}

The strategic alignment among partners in environments with short time horizons, in networks that exploit business opportunities that are limited in time, is an essential reason for companies to use performance evaluation tools and for them to know the degree of compliance/non-compliance with objectives.

Thus, the use of tools for assessment of overall performance can help to achieve operational excellence in dynamic business networks, particularly when the involved partners are heterogeneous concerning management approaches, business culture, etc.

The use of SSM was satisfactory for the implementation of the framework and also for setting and re-setting of performance indicators. In fact, it is appropriate to use 
some methodology in order to create and manage a dynamic performance. It is valid for use on collaborative networks in order to enable to ensure a platform that will facilitate the effectiveness of the strategy.

\section{References}

1. Busi, M., Bititci, U.S.: Collaborative Performance Management: Present Gaps and Future Research. International Journal of Productivity and Performance Management 55(1), 7-25 (2006)

2. Lardenoij, E.J.H., Raaji, E.M., Weele, A.J.: Performance Management Models and Purchasing: Relevance Still Lost. In: Researches in Purchasing and Supply Management. Proceedings, 14th IPSERA Conference, Archamps, pp. 687-697 (2005)

3. Supply Chain Council (SCC): Supply-Chain Operations Reference-Model SCOR: version 9 Overview, http: / / www. supply-chain.org/resources/scor

4. Alba, M., Diez, L., Olmos, E., Rodríguez, R.: Global Performance Management for Small and Medium-sized Enterprises (GMP-SME). In: Camarinha-Matos, L.M., Afsarmanesh, H. (eds.) Collaborative Networks and their Breeding Environments. Springer, New York (2005)

5. Saiz, J.J.A., Rodríguez, R.R., Bas, A.O.: A Performance Measurement System for Virtual and Extended Enterprises. In: Camarinha-Matos, L.M., Afsarmanesh, H. (eds.) Collaborative Networks and their Breeding Environments. Springer, New York (2005)

6. Seifert, M., Wiesner, S., Thoben, K.-D.: Prospective Performance Measurement in Virtual Organisations. In: Camarinha-Matos, L.M., Afsarmanesh, H. (eds.) Collaborative Networks: Reference modeling. Springer, New York (2008)

7. Westphal, I., Thoben, K.-D., Seifert, M.: Measuring Collaboration Performance in Virtual Organisations. Establishing the Foundation of Collaborative Networks. Springer, New York (2007)

8. Azevedo, A.L., Francisco, R.P.: Dynamic Performance Management in Business Networks Environment. Digital Enterprises Technology. Springer, New York (2007)

9. Checkland, P., Scholes, J.: Soft Systems Methodology in action. Wiley, Chichester (1990)

10. Eva, M.: Soft Systems Methodology. Association of Chartered Certified Accountants, http://www.accaglobal.com/students/publications

11. Graelm, F.R., Graelm, K.S., Erdmann, R.H.: Soft Systems Methodology: an Urban Planning Application. In: Proceedings, 6th SIMPOI. FGV, São Paulo (2003)

12. Ferrari, F.M., Fares, C.B., Martinelli, D.P.: The Systemic Approach of SSM: the Case of a Brazilian Company. Systemic Practice and Action Research 15(1), 51-66 (2002)

13. Bergvall-Kareborn, B., Mirijamdotter, A., Basden, A.: Basic Principles of SSM Modeling: an Examination of CATWOE from a Soft Perspective. Systemic Practice and Action Research 17(2), 55-73 (2004)

14. Camarinha-Matos, L.M., Afsarmanesh, H. (eds.): Collaborative Networked Organisations: A Research Agenda for Emerging Business Models. Springer, New York (2004) 